\title{
Cytotoxic effect of Efavirenz in BxPC-3 pancreatic cancer cells is based on oxidative stress and is synergistic with ionizing radiation
}

\author{
MARKUS HECHT $^{1}$, THOMAS HARRER ${ }^{2}$, VERENA KÖRBER ${ }^{1}$, ERIC O. SARPONG ${ }^{1}$, FABIAN MOSER ${ }^{1}$, \\ NORA FIEBIG $^{1}$, MANUELA SCHWEGLER ${ }^{1}$, MICHAEL STÜRZL ${ }^{3}$, RAINER FIETKAU ${ }^{1}$ and LUITPOLD V. DISTEL ${ }^{1}$ \\ Departments of ${ }^{1}$ Radiation Oncology, ${ }^{2}$ Internal Medicine 3 (Infectious Diseases Section) and \\ ${ }^{3}$ Surgery (Division Molecular and Experimental Surgery), Universitätsklinikum Erlangen, \\ Friedrich-Alexander-Universität Erlangen-Nürnberg, D-91054 Erlangen, Germany
}

Received June 14, 2017; Accepted November 20, 2017

DOI: $10.3892 / \mathrm{ol} .2017 .7523$

\begin{abstract}
The non-nucleoside reverse transcriptase inhibitor (NNRTI) Efavirenz is frequently used in human immunodeficiency virus treatment, but also efficient against cancer in mouse models. Its radiosensitizing effect makes it a promising drug for combination with radiotherapy. The efficacy of Efavirenz combined with irradiation was assessed with immunostaining of DNA-damage markers and colony formation assays in BxPC-3 pancreatic cancer cells. Gene expression and protein phosphorylation of the Efavirenz-sensitive BxPC-3 cells was compared to the resistant primary fibroblasts SBL-5. Oxidative stress, mitochondrial damage and cell death were studied with live-cell microscopy and flow cytometry. Combined Efavirenz and radiation significantly increased the number of $\gamma \mathrm{H} 2 \mathrm{AX}$ and phospho-ataxia telangiectasia mutated foci. Efavirenz and ionizing radiation had a synergistic effect using the clonogenic survival assay. Efavirenz selectively induced cell death in the BxPC-3 cells. The differing gene expression of cell cycle and apoptotic regulator genes in both cell cultures after Efavirenz treatment match with this selective effect against cancer cells. In the phosphoprotein array, an early phosphorylation of extracellular signal-related kinase $1 / 2$ and p38 mitogen-activated protein kinase was notably detected in the cancer cells. The phosphorylation of AKT decreased in the cancer cells whereas it increased in the fibroblasts. Oxidative stress and mitochondrial membrane depolarization appeared in the cancer cells immediately after Efavirenz treatment, but not in the fibroblasts. Efavirenz has an anti-cancer effect against pancreatic cancer mainly by the induction of oxidative
\end{abstract}

Correspondence to: Dr Markus Hecht, Department of Radiation Oncology, Universitätsklinikum Erlangen, FriedrichAlexander-Universität Erlangen-Nürnberg, Universitätsstrasse 27, D-91054 Erlangen, Germany

E-mail: markus.hecht@uk-erlangen.de

Key words: Efavirenz, non-nucleoside reverse transcriptase inhibitor, radiation, radiotherapy, pancreatic, cancer, tumor, oxidative, stress stress. The antitumor potential of Efavirenz and radiotherapy are additive.

\section{Introduction}

Non-nucleoside reverse transcriptase inhibitors (NNRTIs) are frequently used in the treatment of HIV-positive patients. However, these drugs also are cytotoxic against different cancer cell lines (1-9). Furthermore, the NNRTI Efavirenz (EFV) has a distinct selectivity against cancer cell lines and spares fibroblast cultures. In this previous analysis toxicity of Efavirenz was studied in three fibroblast and six cancer cell lines with Annexin V-Pi staining and flow cytometry. Efavirenz was solely toxic for cancer cells and spared fibroblasts (9). Treatment with Efavirenz also reduced tumor growth in mouse models $(6,10)$. A recent analysis compared toxicity of six different NNRTIs (Efavirenz, Nevirapine, Rilpivirine, Etravirne, Lersivirine, Delavirdine) against cancer cells and blood levels in HIV-positive patients (8). All six studied NNRTIs became toxic in vitro at different concentrations. When the in vitro toxic concentrations were compared to the blood concentrations of HIV-infected patients, only some patients taking Efavirenz reached the in vitro cytotoxic concentrations. The in vitro cytotoxic concentrations of the other five NNRTIs were never achieved in patients. Consequently, Efavirenz was chosen for the analyses in this study. In a translational approach a radiosensitizing effect of NNRTI, especially Efavirenz, was found in peripheral blood lymphocytes and primary fibroblasts (11). This can lead to an increased radiation-induced toxicity in these patients. If this radiosensitization also appears in cancer cells, the combination of Efavirenz with radiotherapy might improve tumor control. New combination strategies of radiosensitizing agents and radiotherapy may improve future treatment schemes $(12,13)$. These results suggest that Efavirenz may be a promising new drug against cancer either alone or in combination with radiotherapy. Efavirenz has an excellent safety profile compared to classical chemotherapy against cancer (14). This supports the idea to use Efavirenz in cancer patients. We studied the combined effect of Efavirenz with radiotherapy in vitro. Furthermore, specific focus was on the mechanism of Efavirenz induced cell death in pancreatic cancer cells. 


\section{Materials and methods}

Cell culture. The BxPC-3 pancreatic cancer cell line was obtained from the commercial source ATCC (20.02.1997, Salisbury, UK) and grown in Dulbecco's modified Eagle's medium (PAN-Biotech GmbH, Aidenbach, Germany) supplemented with $10 \%$ fetal bovine serum and $1 \%$ penicillin/streptomycin. The primary human fibroblasts SBL-5 were isolated from a healthy donor via skin biopsy of the cutis and subcutis after local anesthesia. The biopsy was dissected in small pieces, placed in tissue culture flasks and each covered with a drop of medium supplemented with $40 \%$ fetal bovine serum. After the skin pieces had attached to the culture flasks and the first fibroblasts had grown out, they were covered with F-12 Medium (Gibco; Live Technologies GmbH, Darmstadt, Germany) supplemented with $12 \%$ fetal bovine serum, $2 \%$ non-essential amino acids and $1 \%$ penicillin/streptomycin. After enough cell divisions of the fibroblasts they were detached with Trypsin and further cultured in the medium mentioned above. Both cell lines were cultured at $37^{\circ} \mathrm{C}$ in a $5 \% \mathrm{CO}_{2}$ incubator.

Drugs. Efavirenz (Sequoia Research Products, Pangbourne, UK) was dissolved in DMSO as $10 \mathrm{mmol} / \mathrm{l}$ stock solution and it was further diluted in medium for use in experiments.

Radiation. Cells were irradiated at a dose rate of 2 Gy per minute with a $120 \mathrm{kV}$ X-ray machine (Isovolt Titan; GE Digital Solutions, Ahrensburg, Germany).

Colony formation assay. At $6 \mathrm{~h}$ after cell seeding, Efavirenz was added. Radiotherapy was delivered after an incubation period of $24 \mathrm{~h}$. Medium containing the drug was removed after a further incubation period of $48 \mathrm{~h}$. The cultures were incubated for three weeks. Colonies were stained with methylene blue for $30 \mathrm{~min}$ at room temperature and clusters containing 50 or more cells were scored as colony. A Zeiss Primo Vert microscope was used with a magnification, $x 100$.

Flow cytometry. Apoptosis and necrosis were detected with APC-labelled Annexin V and 7-Aminoactinomycin (7AAD) as reported before (15). Oxidative Stress was detected with Dihydroethidium (DHE) (Sigma-Aldrich, St. Louis, USA) dissolved in DMSO. Mitochondrial membrane potential was measured with DilC1 (5) (Thermo Fisher Scientific, Waltham, USA). For the combined measurement of apoptosis, oxidative stress and mitochondrial membrane potential a FITC-labelled Annexin V (16) was used. In this experiment DHE was added to the adherent cells for $10 \mathrm{~min}$ (final concentration in the medium was $20 \mu \mathrm{mol} / \mathrm{l}$ ). Afterwards cells were detached with trypsin and washed. They were resuspended in medium containing DilC1 (5) (final concentration in the medium was $15 \mathrm{nmol} / \mathrm{l}$ ) for $15 \mathrm{~min}$. EFV was added if pretreated. Cells were washed again and suspended in ice-cold ringer solution containing Annexin V-FITC for $30 \mathrm{~min}$. After a further washing step, cells were analyzed by a flow cytometer (Gallios Cytometer 1.1 Software; Beckman Coulter GmbH, Krefeld, Germany). Results were analyzed with Kaluza Flow Cytometry Analysis 1.1 (Beckman Coulter $\mathrm{GmbH}$ ).
Immunostaining. Immunostaining was performed as reported before $(11,17)$. Cells were grown on cover slips and incubated with Efavirenz for $24 \mathrm{~h}$ and afterwards irradiated. After further $24 \mathrm{~h}$ for recovery, cells were stained. The following primary antibodies were used: $\gamma \mathrm{H} 2 \mathrm{AX}$ (Ser 139) (cat. no. 05-636; dilution 1:1,500; Merck Milipore, Darmstadt, Germany), phospho-ATM (Ser1981) (cat. no. ab81292; dilution 1:300; Abcam, Cambridge, UK), Ki67 (cat. no. sc-7844; dilution 1:50; Santa Cruz Biotechnology, Inc., Dallas, TX, USA), PML (cat. no. sc-9863; dilution 1:50; Santa Cruz Biotechnology, Inc.). Alexa Fluor 488 (cat. no. A11001; dilution 1:400; Molecular Probes, Eugene, OR, USA) and Alexa Fluor 594 (cat. no. A21442; dilution 1:200; Molecular Probes) were used as secondary antibodies. Incubation time for the primary antibodies was $2 \mathrm{~h}$ at room temperature and for the secondary antibodies $1 \mathrm{~h}$ at room temperature. Greyscale images were captured with a fluorescence microscope (Axioplan 2; Zeiss, Göttingen, Germany; magnification, $\mathrm{x} 400$ ). The number of foci per cell was counted semi-automatically with image analysis software (Biomas Software, Version 3.0; MSAB, Erlangen, Germany).

Life cell imaging. BxPC-3 pancreatic cancer cells were seeded into a microfluidic cell culture chip equipped with two rhombic chambers (microfluidic ChipShop GmbH, Jena, Germany) at a density of approximately $2,5 \times 10^{5}$ cells per chamber. An upright microscope (DM6000; Leica Microsystems GmbH, Wetzlar, Germany) was enclosed by a self-made incubator covering the entire microscope for incubation at $37^{\circ} \mathrm{C}$. At time point zero $20 \mu \mathrm{mol} / 1 \mathrm{DHE}$ was added to one of the chambers and $20 \mu \mathrm{mol} / 1 \mathrm{DHE}$ plus $40 \mu \mathrm{mol} / 1$ Efavirenz was added to the other one. The LAS X module Mark and Find was used to acquire images at three positions in each chamber every $20 \mathrm{sec}$. Images were analyzed by the Biomas image analysis software (version 3.0; MSAB). Cells were marked and the average fluorescence intensity in every cell of the region of interest was transferred to an excel spreadsheet.

Protein array. The PathScan ${ }^{\circledR}$ Stress and Apoptosis Signaling Antibody Array kit (Cell Signaling Technology, Inc., Danvers, MA, USA) was used according to the manufacturer's instructions. The readout was performed with a fluorescence microscope (Axioplan 2; Zeiss).

RNA isolation and quantitative PCR. RNA isolation and quantitative PCR was performed as reported before (18). The following oligonucleotides were designed with Primer 3 and used at 100-300 nmol/1: p27Kip1, forward: 5'-TAACTCTGA GGACACGCATTTG-3', and reverse: 5'-GAGTAGAAGAAT CGTCGGTTGC-3'; Cyclin D1, forward: 5'-GCCTCTAAG ATGAAGGAGACCAT-3', and reverse 5'-AGGTTCCACTTG AGCTTGTTCAC-3'; E2F1, forward: 5'-GTGGACTCTTCG GAGAACTTTCAG-3', and reverse: 5'-AAACATCGATCG GGCCTTGTTTG-3'; Bcl2, forward: 5'-GTGGATGACTGA GTACCTGAACC-3', and reverse: 5'-CTTCAGAGACAGCCA GGAGAAAT-3'; Caspase-8, forward: 5'-TTCTGGAGCATC TGCTGTCTGAGC-3', and reverse: 5'-AGGTTCAAGTGA CCAACTCAAGGG-3'; Orf2, forward: 5'-AAATGGTGC TGGGAAAACTG-3', and reverse: 5'-GCCATTGCTTTT GGTGTTTT-3'; HPRT, forward: 5'-TGCAGACTTTGCTTT 
CCTTGGTC-3', and reverse: 5'-CAAGCTTGCGACCTTGAC CATC-3'; RPUSD3, forward: 5'-AAGACTCTCAGTCACTTT CGTGTG-3', and reverse: 5'-CCTGTAGTTGACTGGAGA ACACTG-3'; TCF20, forward: 5'-GTTACAATGTGAATGC TGGATCTC-3', and reverse: 5'-TTTGCCTGTTCAAAATTC TTCATA-3'; GIGYF2, forward: 5'-GTGCACTAGATGATG AAAGATTGG-3', and reverse: 5'-TGTAATACCACTTCT GCATTGCTT-3'; L3MBTL2, forward: 5'-GAAGGTACG AGCAGTCTACACAGA-3', and reverse: 5'-TCCAGGAGA ACCTTACAGACAGTT-3'.

Optimal reference gene selection and reference target stability were determined with geNorm algorithm (19). As reference genes for the BxPC-3 pancreatic cancer cells the combination of RPUSD3, TCF20 and GIGYF2 and for SBL-5 fibroblasts the combination of GIGYF2 and L3MBTL2 was chosen. The sequence for the primers of Orf2 and its reference gene HPRT1 were chosen as previously published (20). A CFX Real-Time System (Bio-Rad Laboratories, Inc., Hercules, CA, USA) was used and gene expression analysis was performed with CFX Manager Software (Bio-Rad Laboratories, Inc.).

Statistical analysis. All experiments were performed three times. Data analysis was performed with Microsoft Excel 2010 (Microsoft Corporation, Redmond, WA, USA). The Student's t-test was used to test for statistical significance. $\mathrm{P}<0.05$ was considered to indicate a statistically significant difference. Statistical analysis of the protein array was performed with ANOVA and if significant the Tukey's HSD test was used for post hoc analyses. All figures visualize mean values with standard deviation.

\section{Results}

Additive toxicity of Efavirenz and ionizing radiation. Recently, a radiosensitizing effect of NNRTI and especially Efavirenz was detected in ex vivo analyses of human circulating lymphocytes and primary fibroblasts (11). This effect may cause increased radiation-induced adverse events in these patients, but may also improve tumor control. To assess a possibly radiosensitizing effect of Efavirenz in cancer cells, the BxPC-3 pancreatic cancer cell line was treated with Efavirenz $(25 \mu \mathrm{mol} / \mathrm{l})$ and irradiated with $2 \mathrm{~Gy}$. According to previously published drug concentration-response curves of Efavirenz in BxPC-3 cancer cells (8), this slightly toxic drug concentration was chosen for the immunostaining experiments. A double staining of the DNA double-strand break marker $\gamma \mathrm{H} 2 \mathrm{AX}$ (phosphorylated Histone $\mathrm{H} 2 \mathrm{AX}$ ) and the proliferation marker Ki67 was performed (Fig. 1A-D). The number of proliferating Ki67 positive cells clearly decreased when the cells were treated with EFV and especially when they were treated with the combination of EFV and radiation $(\mathrm{P}=0.007$ compared to radiation alone and $\mathrm{P}=0.124$ compared to EFV alone, Fig. 1E). Furthermore, the combination of Efavirenz and radiation increased the number of $\gamma \mathrm{H} 2 \mathrm{AX}$ foci significantly compared to each single agent $(\mathrm{P}=0.008$ compared to radiation alone and $\mathrm{P}=0.028$ compared to EFV alone, Fig. 1F). This increase was apparent both in proliferating (Ki67 positive) and non-proliferating (Ki67 negative) cells, but reached no statistical significance in the subgroups. More repetitions would be necessary reduce the large error bars and confirm the increase of the $\gamma \mathrm{H} 2 \mathrm{AX}$ foci in these subgroups. An increased number of $\gamma \mathrm{H} 2 \mathrm{AX}$ foci indicating DNA damage was found in the proliferating cells. Furthermore, the activation/phosphorylation of the kinase ATM (Ataxia telangiectasia mutated) was studied (Fig. 1G). ATM phosphorylation is a marker of DNA damage repair and cell cycle control. The combination of Efavirenz and radiation increased the number of Ser1981 phosphorylated ATM significantly compared to the single agents $(\mathrm{P}=0.047$ compared to $\mathrm{EFV}$ alone and $\mathrm{P}=0.039$ compared to radiation alone). Finally, the nuclear bodies with its marker protein PML were studied (Fig. 1G). Nuclear bodies are involved in different cellular stress responses. There was a trend to an increased number of PML in the combination of Efavirenz and radiation without reaching statistical significance. The standard assay for radiosensitivity testing is the colony formation assay. In this assay the toxic effect of Efavirenz against pancreatic cancer cells was additive to ionizing radiation (Fig. 1H).

Differential activity of Efavirenz in pancreatic cancer cells and primary fibroblasts. In previous analyses Efavirenz had a distinct selectivity between cancer cells and fibroblasts. Both, cytotoxicity and induction of a G1 cell cycle arrest was increased in cancer cells (9). Cell death was assessed in BxPC-3 pancreatic cancer cells and SBL-5 primary fibroblasts. Flow cytometry with combined Annexin V-APC and 7AAD staining was performed. Annexin $\mathrm{V}$ positive and 7AAD negative cells are classified as apoptotic cells and double positive cells as necrotic cells (Fig. 2A). Efavirenz concentrations of 50 or $60 \mu \mathrm{mol} / 1$ significantly increased cell death in the pancreatic cancer cells after $72 \mathrm{~h}$, whereas the fibroblasts were affected to a much lesser extent. The treatment period of $72 \mathrm{~h}$ was chosen as many chemotherapeutic agents induce a slow cell death response which can last up to $72 \mathrm{~h}$ (21). This treatment period has also been used in previous analyses of Efavirenz induced cell death (9).

Effect of Efavirenz on cell signaling. The effect of Efavirenz on the expression of regulators of cell cycle control and cell death was studied with quantitative PCR. Cells were treated with Efavirenz $40 \mu \mathrm{mol} / 1$ for 3, 6 and $12 \mathrm{~h}$. According to previously published drug concentration-response curves of Efavirenz in BxPC-3 cancer cells (8), this drug concentration is slightly above the EC50 (50\% toxic concentration) was chosen. The normalized relative expression of the cell cycle regulator p27Kip1 clearly increased in the cancer cells (BxPC-3: $12 \mathrm{~h} \mathrm{P=0.038),} \mathrm{whereas} \mathrm{it} \mathrm{decreased} \mathrm{in} \mathrm{the} \mathrm{fibro-}$ blasts (SBL-5) after Efavirenz treatment $(6 \mathrm{~h} \mathrm{P}=0.017$ and $12 \mathrm{~h}$ $\mathrm{P}=0.005$ ) (Fig. 2B and C). The expression of cyclin D1 transiently decreased in both cell types (BxPC-3 $6 \mathrm{~h} \mathrm{P}=0.013$ and SBL-5 $6 \mathrm{~h} \mathrm{P}=0.017$ ). Especially in the cancer cells a reduction of E2F1 expression was observed after treatment (BxPC-3: $3 \mathrm{~h}$ $\mathrm{P}=0.003 ; 6 \mathrm{~h} \mathrm{P}=0.014 ; 12 \mathrm{~h} \mathrm{P}=0.008$ ). The expression of the anti-apoptotic Bcl-2 decreased in the cancer cells $(6 \mathrm{~h} \mathrm{P}=0.006$; $12 \mathrm{~h} \mathrm{P}=0.033$ ), whereas in the fibroblasts an initial decrease (3 h P $=0.020$ ) was followed by a slight and statistically insignificant increase (12 h P=0.101) (Fig. 2D and E). Caspase-8 gene expression diminished in both cell lines (BxPC-3: $6 \mathrm{~h}$ $\mathrm{P}=0.047$ and $12 \mathrm{~h} \mathrm{P}=0.018$; SBL-5: $6 \mathrm{~h} \mathrm{P}=0.019$ ). 

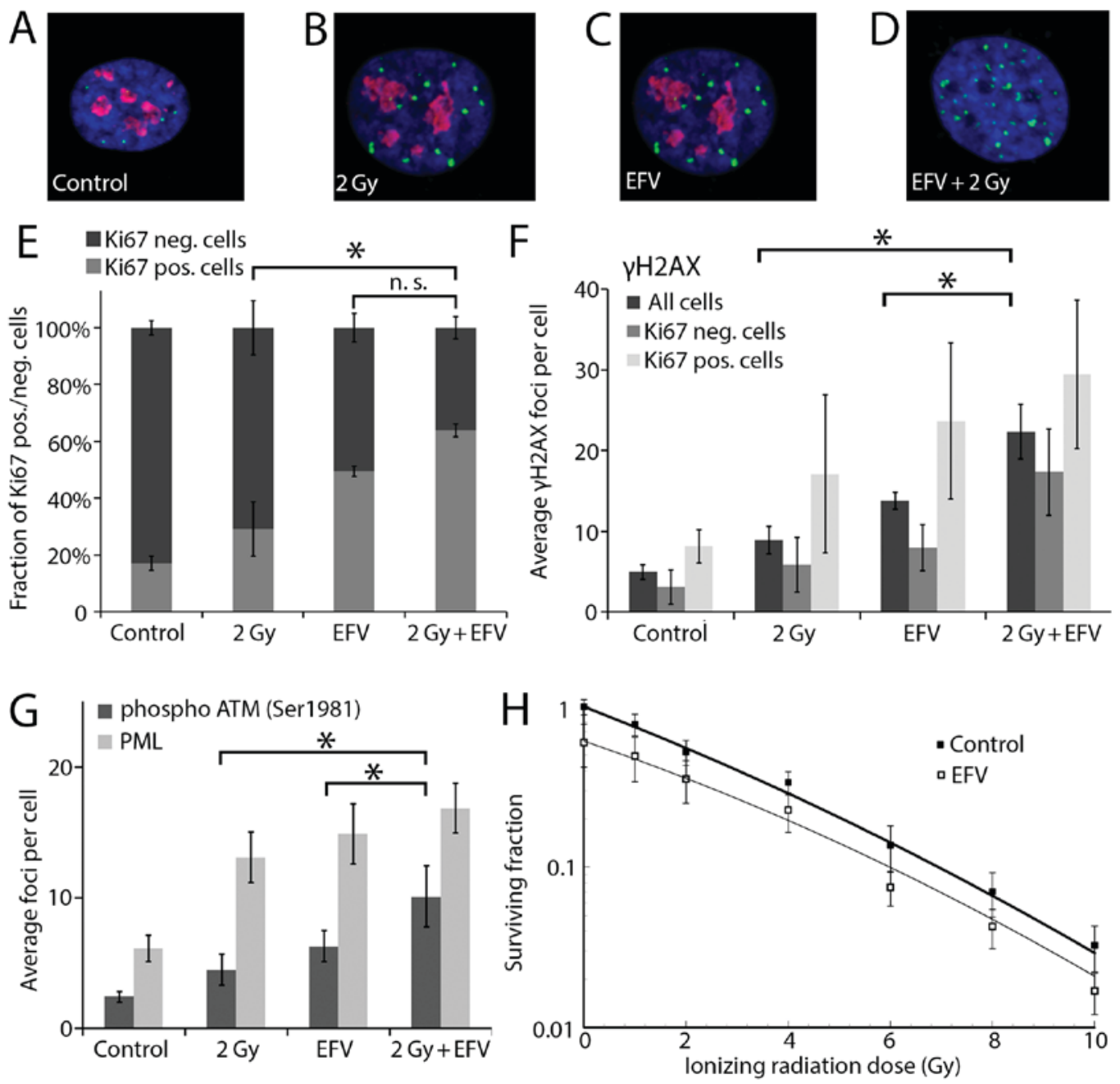

Figure 1. Combination of EFV and irradiation. Each image shows one representative cellular nucleus (blue) with $\gamma \mathrm{H} 2 \mathrm{AX}$ foci (green) and Ki-67 staining (red). (A) One single untreated cellular nucleus of a Ki-67 positive BxPC-3 cell with $\gamma \mathrm{H} 2 \mathrm{AX}$ foci and (B) one irradiated Ki-67 positive cell with $\gamma \mathrm{H} 2 \mathrm{AX}$ foci is shown (24 after radiation). (C) A nucleus with $\gamma \mathrm{H} 2 \mathrm{AX}$ foci in one EFV treated $(25 \mu \mathrm{mol} / 1,48 \mathrm{~h}$ ) Ki-67 positive cell and (D) one irradiated and EFV treated ( $25 \mu \mathrm{mol} / \mathrm{l}$ $24 \mathrm{~h}$ before till $24 \mathrm{~h}$ after radiation) Ki-67 negative cell is shown. (E) Fraction of Ki67 positive/negative cells after treatment with 2 Gy ionizing radiation and EFV ( $25 \mu \mathrm{mol} / 1,24 \mathrm{~h}$ before till $24 \mathrm{~h}$ after irradiation). (F) $\gamma \mathrm{H} 2 \mathrm{AX}$ foci in dependence of Ki-67 in BxPC-3 cancer cells after irradiation and EFV treatment. (G) Phospho-ATM (Ser1981) and PML nuclear bodies after the same treatment. (H) Clonogenic survival (0-10 Gy) with and without EFV. Incubation time was $24 \mathrm{~h}$ before until $24 \mathrm{~h}$ after radiation and colonies were stained after three weeks. "P<0.05. EFV, Efavirenz; PML, promyelocytic leukemia.

Recently, substantial data about a mechanism of action via an NNRTI-sensitive endogenous tumor cell reverse transcriptase were published (20). It was shown that the LINE-1-derived ORF2 product encoded a reverse transcriptase activity. However, no difference in the expression of the respective gene in BxPC-3 and SBL-5 cells was detected (Fig. 2F).

A protein array containing 18 phosphorylated or cleaved proteins was used in order to obtain an overview of putative alterations of different signaling pathways regulating Efavirenz toxicity. BxPC-3 and SBL-5 were treated with Efavirenz $40 \mu \mathrm{mol} / 1$ for $10 \mathrm{~min}, 1$ or $24 \mathrm{~h}$. Fluorescence readout was performed and intensities were corrected with the positive and negative control of the array (Fig. 2G and H). Protein content was normalized to $\alpha$-Tubulin. Especially the early effects were of interest, as these might give some clues to the mechanism of action (Table I). In both cell lines the phosphorylation of both ERK1/2 and p38 MAPK increased at early time points. The phosphorylation of Akt significantly decreased in BxPC-3 cells, whereas it increased in SBL- 5 cells. After $24 \mathrm{~h}$ in the BxPC-3 pancreatic cancer cells a decreased phosphorylation of the anti-apoptotic proteins HSP27, Chk1, TAK1 and Survivin (total protein) was detected. Furthermore, protein levels of phosphorylated Smad2, cleaved Caspase-7, phosphorylated SAPK/JNK and phosphorylated and total IאB $\alpha$ were significantly reduced in the cancer cells after $24 \mathrm{~h}$. In fibroblasts these late effects were not observed.

Oxidative stress induction by Efavirenz. Due to the early increase of the phosphorylation of ERK1/2 and p38 MAPK in both cell lines, oxidative stress was studied as possible mechanism of action (22-24). In Efavirenz induced liver damage oxidative stress also plays an important role (25-29). Reactive oxygen species (ROS) were visualized with DHE and studied in flow cytometric analyses and fluorescence microscopy. In the flow cytometry with the pancreatic cancer cells a shift towards increased DHE staining intensity was detected after Efavirenz treatment compared to the untreated control (Fig. 3A). This effect was not detected in the SBL-5 fibroblasts. In the immunostaining also an increased DHE fluorescence was detected in the BxPC-3 pancreatic cancer cells after 

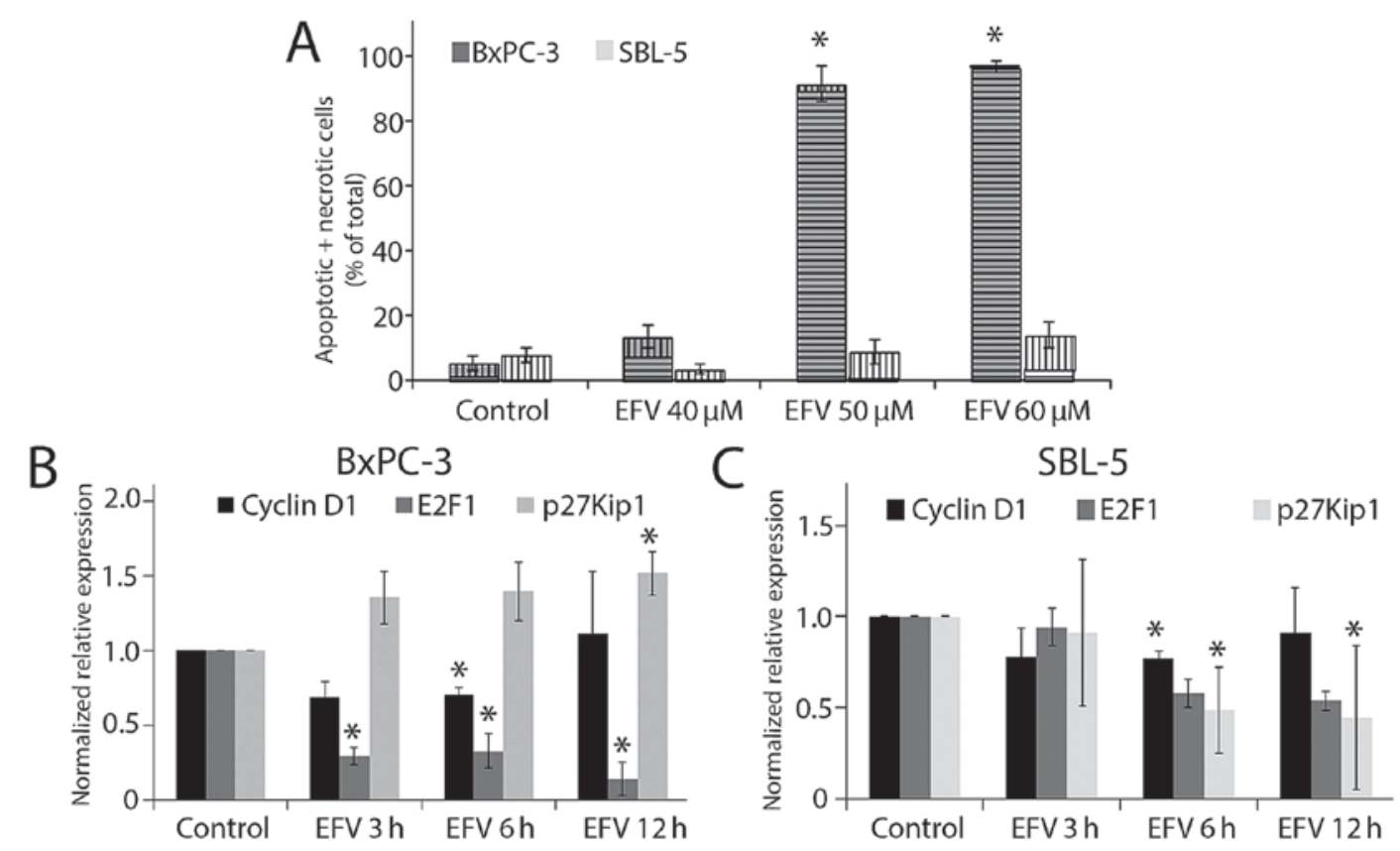
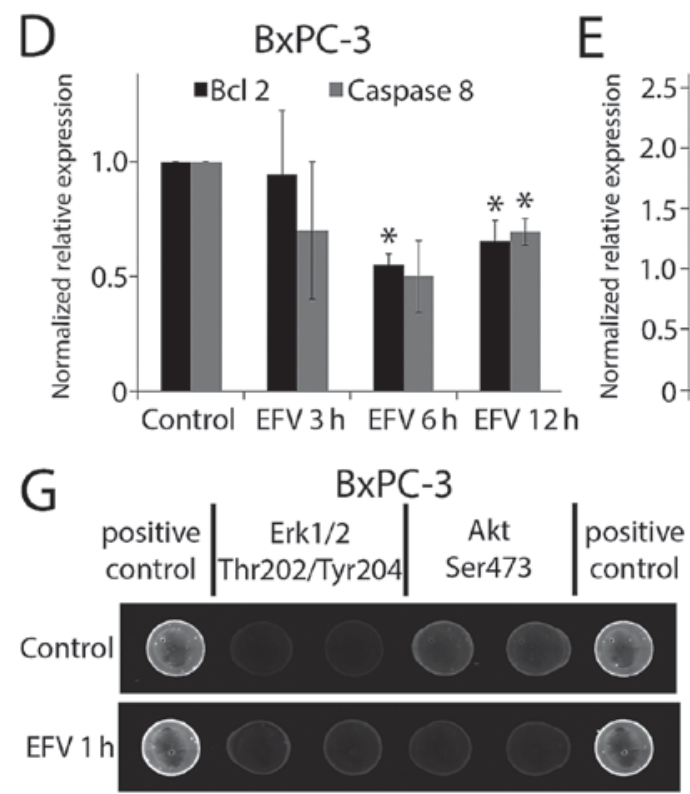
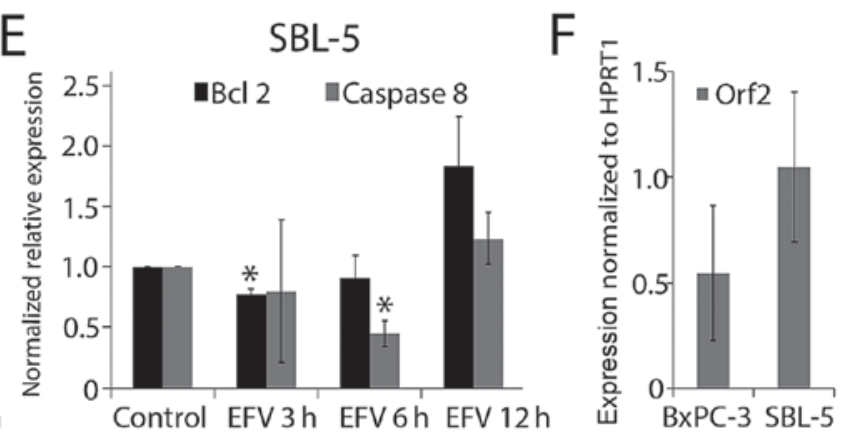

$\mathrm{H}$ \begin{tabular}{c|c|c|c}
$\begin{array}{c}\text { positive } \\
\text { control }\end{array}$ & Thr202/Tyr204 & Ser473 & positive \\
control
\end{tabular}

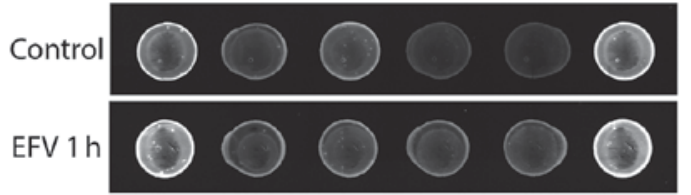

Figure 2. Differential activity of EFV in cancer cells and fibroblasts. BxPC-3 pancreatic cancer cells were compared to SBL-5 primary skin fibroblasts. (A) Effect of EFV treatment for $72 \mathrm{~h}$ on apoptitic death (vertical lines) or necrotic death (horizontal lines) analyzed by flow cytometry after Annexin/7AAD staining. Expression of cell cycle regulators Cyclin D1, E2F1 and p27Kip1 after EFV (40 $\mu \mathrm{mol} / 1)$ treatment on (B) BxPC-3 and (C) SBL-5 cells. Expression of anti-apoptotic signal BCL 2 and Caspase 8 after EFV ( $40 \mu \mathrm{mol} / 1)$ treatment on (D) BxPC-3 and (E) SBL-5 cells. (F) Reference genes for the BxPC-3 pancreatic cancer cells were the combination of RNA pseudouridylate synthase domain containing 3, transcription factor 20 and GIGYF2 and for SBL-5 fibroblasts the combination of GIGYF2 and L3MBTL2 polycomb repressive complex 1 subunit. Expression of LINE1-encoded endogenous reverse transcriptase open reading frame 2. Reference gene for the comparison was HPRT1. Example of the spots of the PathScan ${ }^{\circledR}$ Stress and Apoptosis Signaling Antibody Array in the fluorescence readout for (G) BxPC-3 and (H) SBL-5. "P<0.05. GIGYF2, GRB10 interacting GYF protein 2; EFV, Efavirenz.

Efavirenz treatment, but not in the SBL-5 fibroblasts (Fig. 3B). Life cell imaging with BxPC-3 cells and DHE staining was used to study kinetics of the oxidative stress induction. Oxidative stress appeared immediately after Efavirenz treatment and increased rapidly especially during the first hour (Fig. 3C). In a multi-color flow cytometric assay oxidative stress (DHE), mitochondrial membrane potential (DilC) and apoptosis (Annexin V) were measured simultaneously. Oxidative stress and mitochondrial depolarization appeared immediately after addition of Efavirenz (Fig. 3D). After $48 \mathrm{~h}$ the cell death marker Annexin V was detectable.

\section{Discussion}

Efavirenz is a frequently used drug in HIV-1 treatment. Its safety profile allows a long-term treatment (14). Recently, an increased individual radiosensitivity was found in HIV-1-infected patients treated with NNRTIs (11). This patient blood analysis included both patients with Efavirenz and Nevirapine. These results were confirmed in vitro in Efavirenz treated primary fibroblasts (11). This is probably the reason for the increased rate of adverse events in HIV-infected patients treated with radiotherapy. However, this radiosensitizing effect might also 


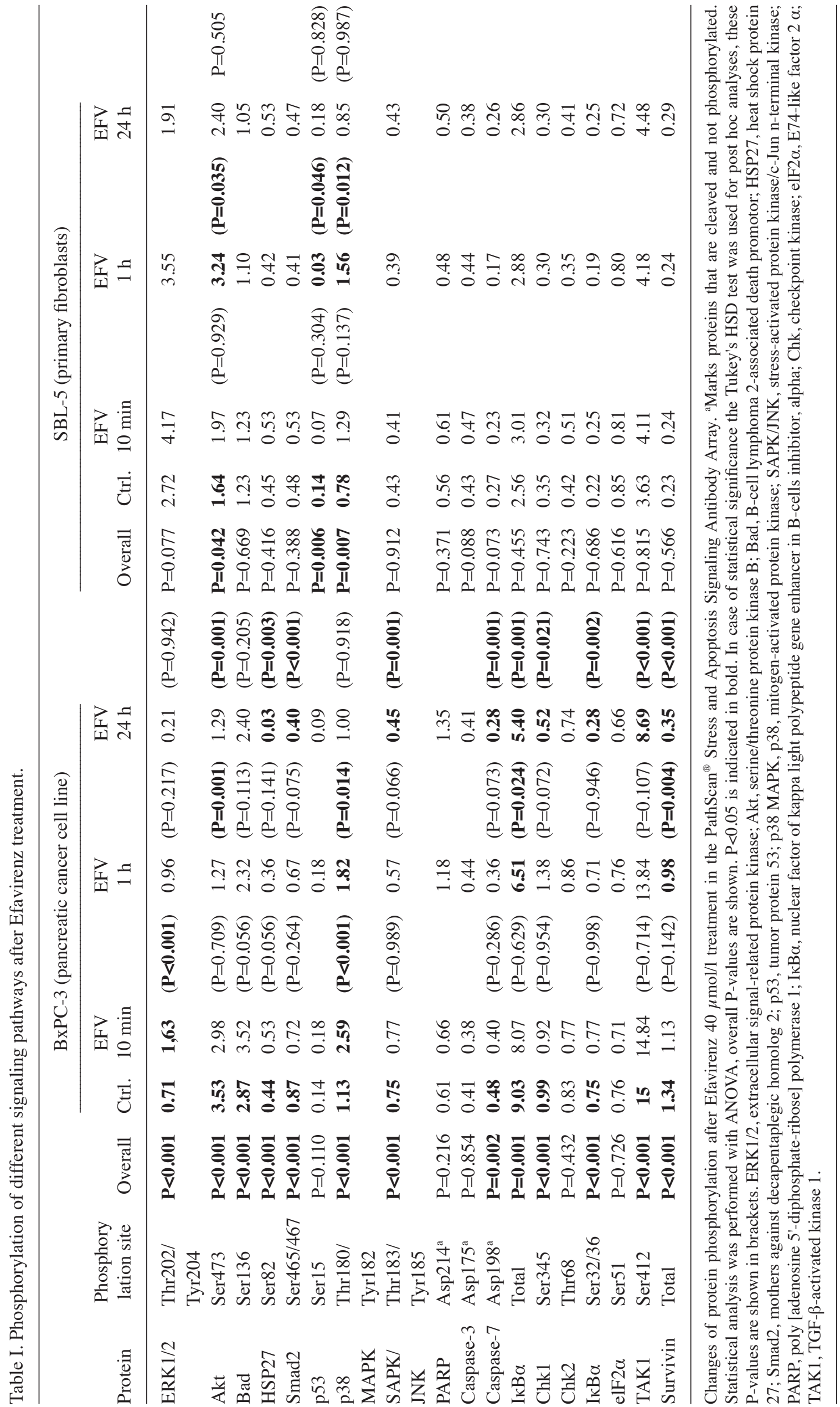



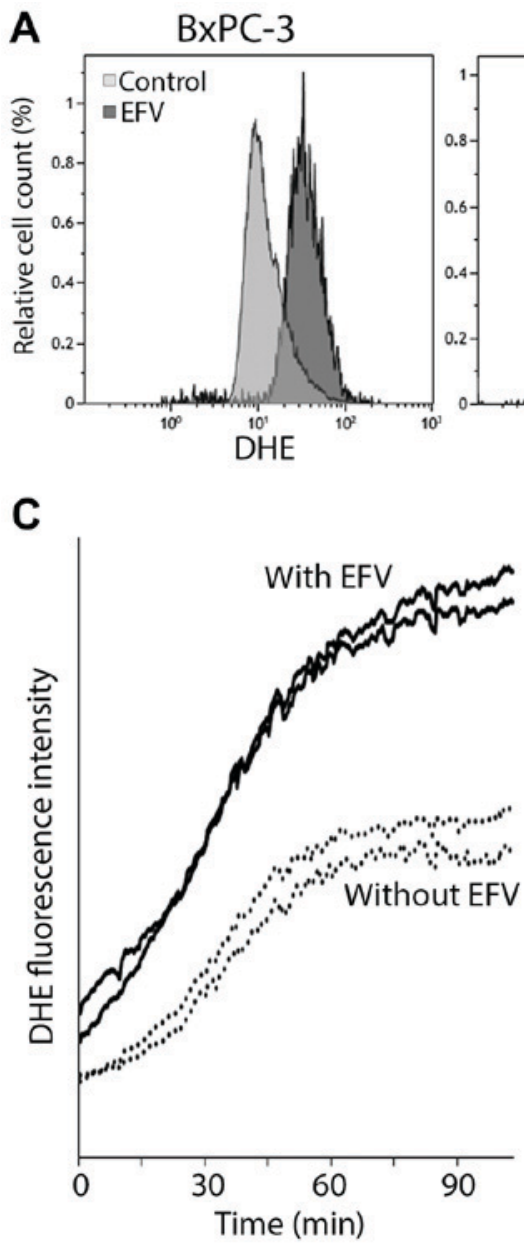

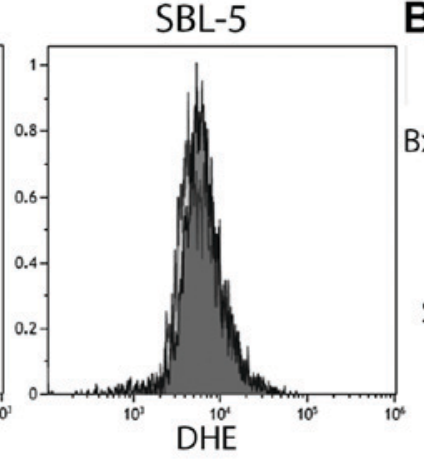

B

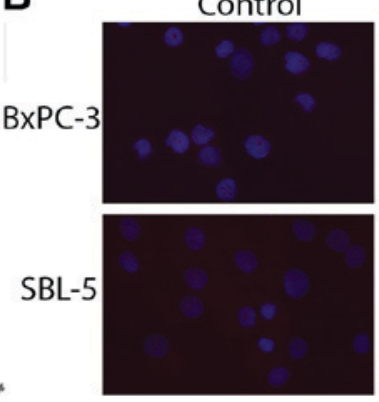

EFV

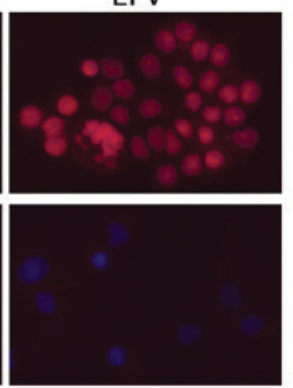

D

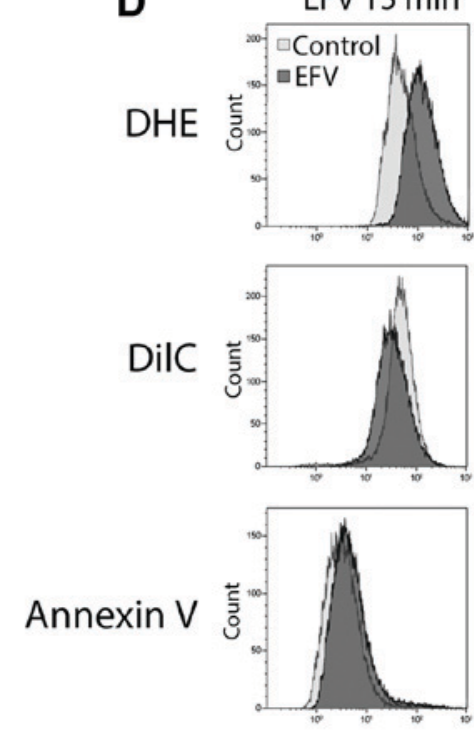

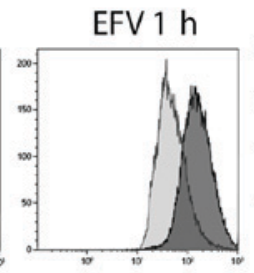
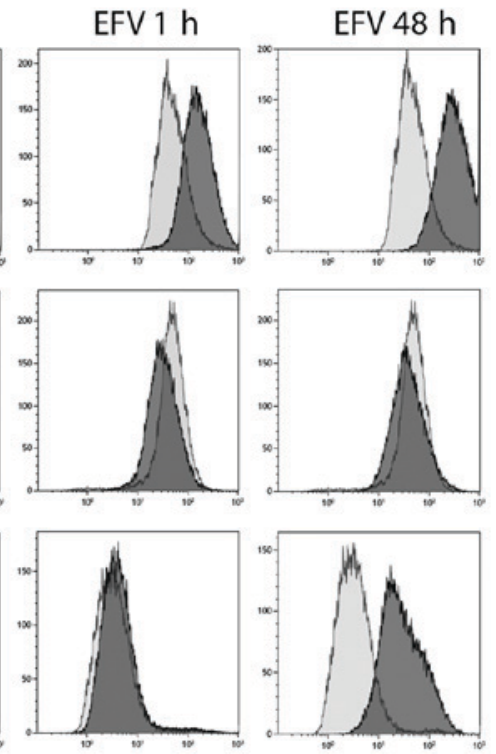

Figure 3. EFV-induced oxidative stress. (A) Flow cytometric assessment and (B) immunostaining of oxidative stress induction with DHE in BxPC-3 pancreatic cancer cells and SBL-5 primary fibroblasts treated with EFV (40 $\mu$ mol/1 for $1 \mathrm{~h})$. (C) Time kinetics of oxidative stress induction after EFV treatment (40 $\mu \mathrm{mol} / \mathrm{l})$ in life cell imaging in BxPC-3 cells. Fluorescence intensity was scanned at two positions with EFV (solid lines) and two positions without EFV (dotted lines). (D) Simultaneous flow cytometric assessment of oxidative stress (DHE), mitochondrial membrane potential (DilC) and apoptosis (Annexin V) after EFV treatment. DHE, Dihydroethidium; EFV, Efavirenz.

sensitize cancer cells and consequently improve tumor control. In the colony formation assay with BxPC-3 pancreatic cancer cells, Efavirenz had an additive effect in combination with radiotherapy. The combination of Efavirenz with ionizing radiation also increased the rate of DNA-double strand breaks marked by $\gamma \mathrm{H} 2 \mathrm{AX}$, especially in proliferating cells. These results are in line with the increased activation of the kinase ATM that is essential for DNA-damage repair and cell cycle control (30). In conclusion, the combination of Efavirenz with radiotherapy has synergistic activity against pancreatic cancer cells. It is suspected that this synergistic toxicity also appears when other NNRTIs are combined with radiotherapy.

Several in vitro studies demonstrated that NNRTIs are toxic against cancer cells even without radiotherapy (1-9). In these studies Efavirenz was toxic against cell lines consisting of renal carcinoma, prostate carcinoma, anaplastic thyroid carcinoma, sarcoma, colorectal carcinoma, breast carcinoma, glioblastoma, cervical carcinoma, pancreatic carcinoma and head and neck squamous cell carcinoma. This finding was confirmed with mice experiments in vivo $(6,10)$. Nude mice were inoculated with tumor cells and treated with Efavirenz. Efavirenz treatment led to a significant growth reduction of the tumor compared to untreated mice. An important question is if the toxic Efavirenz concentrations in vitro or in mice experiments can be reached in humans. In a previous analysis the in vitro zytotoxic concentrations of six different NNRTIs were compared to blood levels of HIV-infected patients. Only some patients taking Efavirenz reached the in vitro toxic concentrations of Efavirenz (8). The in vitro toxic concentrations of other NNRTIs have not been reached in HIV-infected patients so far. A distinct selectivity of Efavirenz toxicity against pancreatic cancer cells compared to fibroblasts has already been described (9) and is the basis for the mechanistic studies of this work. In the flow cytometric assessment of cell death, Efavirenz was toxic against BxPC-3 pancreatic cancer cells, but not human fibroblasts. A limitation of these experiments is the lacking positive control, which should be added in future experiments. The expression of different important cell cycle and cell death regulators differed between the Efavirenz sensitive pancreatic cancer cells BxPC-3 and the primary fibroblasts SBL-5. It has previously been described that Efavirenz induces a G0/G1 arrest in cancer cells $(3,6,9)$. The increased expression of p27Kip1 and reduced expression of cyclin D1 and especially E2F1 (31-33) is in line with this cell cycle arrest. The decreased expression of the anti-apoptotic BCL2 in the cancer cells is probably an early effect of the upcoming cell death (34). 
A protein array was used to screen for the mechanism of action. Especially the early effects on the array are of major interest, as they may give hints to the primary affected signaling pathways. In both cell lines an early phosphorylation of ERK1/2 and p38 MAPK was found, whereas the expression of AKT increased in the fibroblasts but decreased in the cancer cells. AKT is known to play a pivotal role in regulation of cellular survival processes and is a promising target in the treatment of pancreatic cancer (35). The increased phosphorylation of AKT in fibroblasts, is probably the key survival signal for these cells (36). There were several late changes of many other proteins including HSP27, Chk1, TAK1 and Survivin in the cancer cells. These signals are probably consequences from the primary signaling and indicators of an upcoming cell death. The parallel early activation of the stress activated pathways ERK1/2 and p38 MAPK prompted us to study oxidative stress (22-25).

Efavirenz induced reactive oxygen species (ROS) in the BxPC-3 pancreatic cancer cells, but not in fibroblasts. This finding is in line with the mechanism of Efavirenz induced liver damage, which is also induced by oxidative stress and caused by mitochondrial damage (25-29). Since both liver cells and cancer cells harbor an increased number of mitochondria, mitochondrial impairment might be causative. Further research is required to verify the oxidative stress induction in cancer cells and characterize its origin e.g., with mitochondria-specific superoxide sensors. In liver toxicity an inhibition of complex 1 of the mitochondrial respiratory chain is suspected (27-29). The rapid onset of oxidative stress after Efavirenz treatment supports this direct mitochondrial toxicity and argues against a mechanism based on altered genetic expression after inhibition of an endogenous reverse transcriptase. This published mechanism suspects an endogenous LINE-1-encoded reverse transcriptase, which is expressed in cancer cells and can be inhibited by NNRTIs $(2-6,10,37)$. The expression of this endogenous reverse transcriptase did not differ in the fibroblasts and the pancreatic cancer cells. However, these two published mechanisms might be dose dependent. It is possible that long-term low-dose Efavirenz treatment might inhibit this tumor reverse transcriptase and lead to genetic reprogramming, whereas cytotoxicity is mediated via oxidative stress when higher Efavirenz doses are used.

Data about clinical efficacy of NNRTIs in cancer patients are currently still rare. The other approved first-generation NNRTI Nevirapine was used in single HIV-negative patients with poorly differentiated thyroid cancer. It induced cell differentiation of the poorly differentiated thyroid cancer and restored iodine-131 uptake in the cancer cells $(38,39)$. In HIV-infected patients regression of lymphomas was described after Efavirenz based antiretroviral treatment $(40,41)$. In a phase II clinical trial in 53 patients with metastatic castration-resistant prostate cancer a non-progression rate of $28 \%$ was observed after 3 months (42). Efavirenz plasma concentrations above $3,000 \mathrm{ng} / \mathrm{ml}$ were associated with a reduced risk of progression. Based on the in vitro findings of this work, prostate cancer seems to be not a very suitable type of cancer for Efavirenz treatment due to its slow growth kinetics. A better treatment response to Efavirenz might be possible in faster growing cancers with a higher metabolism and subsequently stronger dependence on mitochondrial function.
Efavirenz and radiotherapy have a synergistic toxic effect against pancreatic cancer. The reported results shed light on the cytotoxic effect of Efavirenz by induction of oxidative stress in the studied pancreatic cancer cell line. Efavirenz toxicity was shown to be selective against cancer cells to some extent. Potential role of AKT signaling for cell death of cancer cells and survival of normal fibroblasts should be examined in future experiments to bring further insight into Efavirenz-related tumor cell death.

\section{Acknowledgements}

Markus Hecht was funded by the ELAN-Fonds (15-02-16-1-Hecht). The authors want to thank the committee of the ELAN-Fonds of the Friedrich-Alexander-Universität Erlangen-Nürnberg for funding this project. $\mathrm{MH}$ reports funding for research from Novartis and MSD, honoraria from BMS, travelling support from Merck Serono and MSD (all outside this project). TH reports funding for research from Abbott and Janssen, honoraria from BMS, Böhringer, GSK, Viiv, Abbott Jansen-Tibotec, Gilead, Essex and Janssen (all outside this project). RF reports research funding for research from MSD, honoraria from Roche, Merck Serono, Fresenius and MSD, travelling support from Roche, Merck Serono, Fresenius and MSD (all outside this project).

\section{References}

1. Landriscina M, Altamura SA, Roca L, Gigante M, Piscazzi A Cavalcanti E, Costantino E, Barone C, Cignarelli M, Gesualdo L and Ranieri E: Reverse transcriptase inhibitors induce cell differentiation and enhance the immunogenic phenotype in human renal clear-cell carcinoma. Int J Cancer 122: 2842-2850, 2008.

2. Landriscina M, Bagalá C, Piscazzi A, Schinzari G, Quirino M, Fabiano A, Bianchetti S, Cassano A, Sica G and Barone C: Nevirapine restores androgen signaling in hormone-refractory human prostate carcinoma cells both in vitro and in vivo. Prostate 69: 744-754, 2009.

3. Landriscina M, Fabiano A, Altamura S, Bagalà C, Piscazzi A, Cassano A, Spadafora C, Giorgino F, Barone C and Cignarelli M: Reverse transcriptase inhibitors down-regulate cell proliferation in vitro and in vivo and restore thyrotropin signaling and iodine uptake in human thyroid anaplastic carcinoma. J Clin Endocrinol Metab 90: 5663-5671, 2005.

4. Mangiacasale R, Pittoggi C, Sciamanna I, Careddu A, Mattei E, Lorenzini R, Travaglini L, Landriscina M, Barone C, Nervi C, et al: Exposure of normal and transformed cells to nevirapine, a reverse transcriptase inhibitor, reduces cell growth and promotes differentiation. Oncogene 22: 2750-2761, 2003.

5. Pittoggi C, Martis G, Mastrangeli G, Mastrangeli B and Spadafora C: In vitro evidence for a new therapeutic approach in renal cell carcinoma. Int Braz J Urol 34: 492-502, 2008.

6. Sciamanna I, Landriscina M, Pittoggi C, Quirino M, Mearelli C, Beraldi R, Mattei E, Serafino A, Cassano A, Sinibaldi-Vallebona $\mathrm{P}$, et al: Inhibition of endogenous reverse transcriptase antagonizes human tumor growth. Oncogene 24: 3923-3931, 2005.

7. Stefanidis K, Loutradis D, Vassiliou LV, Anastasiadou V, Kiapekou E, Nikas V, Patris G, Vlachos G, Rodolakis A and Antsaklis A: Nevirapine induces growth arrest and premature senescence in human cervical carcinoma cells. Gynecol Oncol 111: 344-349, 2008.

8. Hecht M, Erber S, Harrer T, Klinker H, Roth T, Parsch H, Fiebig N, Fietkau R and Distel LV: Efavirenz has the highest anti-proliferative effect of non-nucleoside reverse transcriptase inhibitors against pancreatic cancer cells. PLoS One 10: e0130277, 2015.

9. Hecht M, Harrer T, Büttner M, Schwegler M, Erber S, Fietkau R and Distel LV: Cytotoxic effect of efavirenz is selective against cancer cells and associated with the cannabinoid system. AIDS 27: 2031-2040, 2013. 
10. Sinibaldi-Vallebona P, Lavia P, Garaci E and Spadafora C: A role for endogenous reverse transcriptase in tumorigenesis and as a target in differentiating cancer therapy. Genes Chromosomes Cancer 45: 1-10, 2006.

11. Ulrike K, Markus H, Thomas H, Ellen H, Barbara S, Rainer F and Distel LV: NNRTI-based antiretroviral therapy may increase risk of radiation induced side effects in HIV-1-infected patients. Radiother Oncol 116: 323-330, 2015.

12. Baues C, Semrau R, Gaipl US, Bröckelmann PJ, Rosenbrock J, Engert A and Marnitz S: Checkpoint inhibitors and radiation treatment in Hodgkin's lymphoma: New study concepts of the German Hodgkin Study Group. Strahlenther Onkol 193: 95-99, 2017.

13. Schulze B, Meissner M, Ghanaati S, Burck I, Rödel C and Balermpas P: Hedgehog pathway inhibitor in combination with radiation therapy for basal cell carcinomas of the head and neck: First clinical experience with vismodegib for locally advanced disease. Strahlenther Onkol 192: 25-31, 2016.

14. van den Berg-Wolf M, Hullsiek KH, Peng G, Kozal MJ, Novak RM, Chen L, Crane LR and Macarthur RD; CPCRA 058 Study Team, the Terry Beirn Community Programs for Clinical Research on AIDS (CPCRA), and The International Network for Strategic Initiative in Global HIV Trials (INSIGHT): Virologic, immunologic, clinical, safety, and resistance outcomes from a long-term comparison of efavirenz-based versus nevirapine-based antiretroviral regimens as initial therapy in HIV-1-infected persons. HIV Clin Trials 9: 324-336, 2008.

15. Winkler S, Hoppe P, Haderlein M, Hecht M, Fietkau R and Distel LV: Ex vivo apoptosis in CD8+ lymphocytes predicts rectal cancer patient outcome. Gastroenterol Res Pract 2016: 5076542, 2016 .

16. Kotter B, Frey B, Winderl M, Rubner Y, Scheithauer H, Sieber R, Fietkau R and Gaipl US: The in vitro immunogenic potential of caspase-3 proficient breast cancer cells with basal low immunogenicity is increased by hypofractionated irradiation. Radiat Oncol 10: 197, 2015

17. Endt H, Sprung CN, Keller U, Gaipl U, Fietkau R and Distel LV: Detailed analysis of DNA repair and senescence marker kinetics over the life span of a human fibroblast cell line. J Gerontol A Biol Sci Med Sci 66: 367-375, 2011.

18. Schwegler M, Wirsing AM, Dollinger AJ, Abendroth B, Putz F, Fietkau R and Distel LV: Clearance of primary necrotic cells by non-professional phagocytes. Biol Cell 107: 372-387, 2015.

19. Vandesompele J, De Preter K, Pattyn F, Poppe B, Van Roy N, De Paepe A and Speleman F: Accurate normalization of real-time quantitative RT-PCR data by geometric averaging of multiple internal control genes. Genome Biol 3: RESEARCH0034, 2002.

20. Patnala R, Lee SH, Dahlstrom JE, Ohms S, Chen L, Dheen ST and Rangasamy D: Inhibition of LINE-1 retrotransposon-encoded reverse transcriptase modulates the expression of cell differentiation genes in breast cancer cells. Breast Cancer Res Treat 143: 239-253, 2014

21. Blois J, Smith A and Josephson L: The slow cell death response when screening chemotherapeutic agents. Cancer Chemother Pharmacol 68: 795-803, 2011.

22. Banno Y, Wang S, Ito Y, Izumi T, Nakashima S, Shimizu T and Nozawa Y: Involvement of ERK and p38 MAP kinase in oxidative stress-induced phospholipase D activation in PC12 cells. Neuroreport 12: 2271-2275, 2001.

23. Matos TJ, Duarte CB, Gonçalo M and Lopes MC: Role of oxidative stress in ERK and p38 MAPK activation induced by the chemical sensitizer DNFB in a fetal skin dendritic cell line. Immunol Cell Biol 83: 607-614, 2005.

24. Son Y, Cheong YK, Kim NH, Chung HT, Kang DG and Pae HO Mitogen-activated protein kinases and reactive oxygen species: How can ROS activate MAPK pathways? J Signal Transduct 2011: 792639, 2011.

25. Apostolova N, Gomez-Sucerquia LJ, Alegre F, Funes HA, Victor VM, Barrachina MD, Blas-Garcia A and Esplugues JV: ER stress in human hepatic cells treated with Efavirenz: mitochondria again. J Hepatol 59: 780-789, 2013.
26. Apostolova N, Gomez-Sucerquia LJ, Gortat A, Blas-Garcia A and Esplugues JV: Compromising mitochondrial function with the antiretroviral drug efavirenz induces cell survival-promoting autophagy. Hepatology 54: 1009-1019, 2011.

27. Apostolova N, Gomez-Sucerquia LJ, Moran A, Alvarez A, Blas-Garcia A and Esplugues JV: Enhanced oxidative stress and increased mitochondrial mass during efavirenz-induced apoptosis in human hepatic cells. Br J Pharmacol 160: 2069-2084, 2010.

28. Blas-Garcia A, Apostolova N, Ballesteros D, Monleón D, Morales JM, Rocha M, Victor VM and Esplugues JV: Inhibition of mitochondrial function by efavirenz increases lipid content in hepatic cells. Hepatology 52: 115-125, 2010.

29. Gomez-Sucerquia LJ, Blas-Garcia A, Marti-Cabrera M, Esplugues JV and Apostolova N: Profile of stress and toxicity gene expression in human hepatic cells treated with Efavirenz. Antiviral Res 94: 232-241, 2012.

30. Shiloh Y and Ziv Y: The ATM protein kinase: Regulating the cellular response to genotoxic stress and more. Nat Rev Mol Cell Biol 14: 197-210, 2013

31. Baldin V, Lukas J, Marcote MJ, Pagano M and Draetta G: Cyclin $\mathrm{D} 1$ is a nuclear protein required for cell cycle progression in G1. Genes Dev 7: 812-821, 1993.

32. Johnson DG, Schwarz JK, Cress WD and Nevins JR: Expression of transcription factor E2F1 induces quiescent cells to enter $\mathrm{S}$ phase. Nature 365: 349-352, 1993.

33. Polyak K, Lee MH, Erdjument-Bromage H, Koff A, Roberts JM, Tempst $\mathrm{P}$ and Massagué J: Cloning of p27Kip1, a cyclin-dependent kinase inhibitor and a potential mediator of extracellular antimitogenic signals. Cell 78: 59-66, 1994.

34. Hatok $\mathrm{J}$ and Racay P: Bcl-2 family proteins: Master regulators of cell survival. Biomol Concepts 7: 259-270, 2016.

35. Ebrahimi S, Hosseini M, Shahidsales S, Maftouh M, Ferns GA, Ghayour-Mobarhan M, Hassanian SM and Avan A: Targeting the Akt/PI3K signaling pathway as a potential therapeutic strategy for the treatment of pancreatic cancer. Curr Med Chem: 24 , 1321-1331, 2017.

36. Manning BD and Cantley LC: AKT/PKB signaling: Navigating downstream. Cell 129: 1261-1274, 2007.

37. Sciamanna I, Gualtieri A, Cossetti C, Osimo EF, Ferracin M, Macchia G, Aricò E, Prosseda G, Vitullo P, Misteli T and Spadafora C: A tumor-promoting mechanism mediated by retrotransposon-encoded reverse transcriptase is active in human transformed cell lines. Oncotarget 4: 2271-2287, 2013

38. Landriscina M, Modoni S, Fabiano A, Fersini A, Barone C, Ambrosi A and Cignarelli M: Cell differentiation and iodine-131 uptake in poorly differentiated thyroid tumour in response to nevirapine. Lancet Oncol 7: 877-879, 2006.

39. Modoni S, Landriscina M, Fabiano A, Fersini A, Urbano N, Ambrosi A and Cignarelli M: Reinduction of cell differentiation and 131I uptake in a poorly differentiated thyroid tumor in response to the reverse transcriptase (RT) inhibitor nevirapine. Cancer Biother Radiopharm 22: 289-295, 2007.

40. Amengual JE, Zhang X, Ibrahim S and Gardner LB: Regression of HIV-related diffuse large B-cell lymphoma in response to antiviral therapy alone. Blood 112: 4359-4360, 2008.

41. Girard T, Luquet-Besson I, Baran-Marszak F, Raphael M and Boue F: HIV+ MALT lymphoma remission induced by highly active antiretroviral therapy alone. Eur J Haematol 74: 70-72, 2005.

42. Houédé N, Pulido M, Mourey L, Joly F, Ferrero JM, Bellera C, Priou F, Lalet C, Laroche-Clary A, Raffin MC, et al: A phase II trial evaluating the efficacy and safety of efavirenz in metastatic castration-resistant prostate cancer. Oncologist 19: 1227-1228, 2014.

This work is licensed under a Creative Commons Attribution 4.0 International (CC BY 4.0) License. 